\title{
MORTALIDAD NEONATAL INTRAHOSPITALARIA:
}

\author{
HALAZGOS ANATOMO - PATOLOGICOS *
}

\author{
Di. NICUTAS HSFINRSA, DE. CAFLOS NALANJO y Dr. JORGF, BUENO

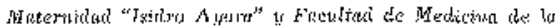

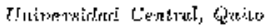

Lín la Maternidad "Isidro Ayora" de Quito, se realizan algo más thel 50 ; the los nacimientos de la ciudad. Alrede. tior de las $\%$ partes de las gestantes que se atierder er etic Servicio IIuspitalarjo, pertenecen a silit genteral, un $15 \%$ sun petsjondstas o scmipensionistris $y$ alrededor de un $10 \%$ sur afiliaclus us Stzyro Sucial !.

Disde el año 1959 basta 1963 se han procluciclo en tutal 14.939 nanimiencos con virla, distribuídus asutulmente de la siguiente monera: 1959, 7.036; 1960, 6.388; 1961, 7.229; 1962, 6.591; 1963, 7.295 .

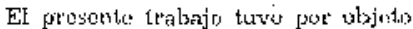
realizar un estudip exhaustivo de lus tullaysos amatomopatológicos en riqui-

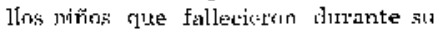
sitadía en mlitstro servicio. Los indices de mortalidad neonatal in(rullospitalaria (29 por uril) y mortalidax perina[a] (49 por tril) observiados durante cstos

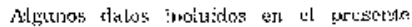

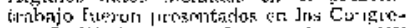

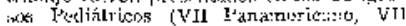

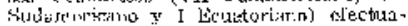

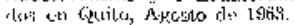

mismos anos, se [istudiän y anali\%an otro trabajo ${ }^{2}$. Trebe cutaizarse subre lil alta imeidencia de infectiones tomo cousa de raxulkta mensatal dobida, en jacte, al proncdimjento equivacadis the autorizar ell ingreses do siños monores de um año de edod, que vienen de afuera con infecciontes; a los Scrvicios de lat vididernitiad, en donde al ser atendir.jers pior un persorial escedsó tin múmero y mal proparado, disconinan lales it!feceiones a jos otros niños.

Los ballazkos qute se presenlaji, laastios en Jes autopijts realizadas, nos permiten apreciar la torma cingo nuestro Departamento fumeiones, anulizar Jas calusas de la mortalidad nematial $y$ Ja predexencia rue tiente nueshro porsonal para restizar eicrto tijuo de autiopm sia.s.

\section{WATHRIALES Y MLYODOS}

Se had realizado rstudios postmosten en un $30 \%$ do los nin̄es fallecidos. En ucusiones, fror insxpmeriencis de

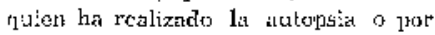


lalta de diempo y otras difieultades, los estudios heehos no han sido sistemáticos y esmpletos.

Con frecuercia, ste efectuaron las atdionsiä en cudáveres mal comservados, cuando había transcurrido un tiempo de: mucte mayor de 24 boras, hista loculizat a los familares y consegraj aulorización para los exámenes postmorten.

Ninstev personal hat demostrado $\lll-$ jecial empeño sur conseguit' autopsits. de It's eduro que le han porreciclo més interesantes; do se ha tonldn en ramhìc al misuru interés para bacer estudios postmorten de los permaturos muertos ton sindrome de dificultad respiratoria, $\mathbb{E}, \dot{s}$ por elli ryue la fre- cuencia de altupsias on los cases de sitilis congéniliz $y$ malformaciones congínitas, que ham despertado un interés mayor, es más torande que la incidencia real de esa patología y etr carm.bjı sucede Io cuntrarits em el síndrume de dif́icullat res]zirutoria del prematuro.

So han exclujo de esie trabijo pocas dutopsias que no han terides el mínimo de datos que "pel'mi lian haces' lo sis" tematizacion gue sus hernos propuesto; on usos casoi no hemos podido encortrur parcial o toilmente la información nesesarja de las historias clínicas o de los cotudios jusstmurter.

Hi estudin macroscópico die las pie7as hil sirln incompleto y realizntio a venes por el interno del Sorvicio, sin

LABLA I

CAUSAS ANATOMTCAS DH MLLRTE OBTENIDAS WN LA MATHRINDAI "ISHTRO AYORA" DE QUUTO, COMPARADAS CON LAS DE SERVICIOS PJDIN'I'RICOS DE ESIADOS UNEDOS I"

\begin{tabular}{|c|c|c|c|c|c|c|}
\hline \multirow[t]{2}{*}{ Jlrthagges postimurdar! } & \multirow[t]{2}{*}{$\begin{array}{c}\text { Chicage } \\
\psi_{1}\end{array}$} & \multirow[t]{2}{*}{$\begin{array}{l}\text { Yew } \\
\text { Yorl; } \\
\%\end{array}$} & \multirow[t]{2}{*}{$\begin{array}{c}\text { London } \\
\text { Hosprial } \\
\text { \% }\end{array}$} & \multirow[t]{2}{*}{ 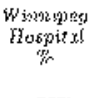 } & \multicolumn{2}{|c|}{ 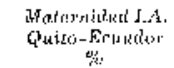 } \\
\hline & & & & & $A^{\circ}$ & $13: 4$ \\
\hline Vorelilacion pulmonar omphenal .. & 47,3 & दid & 20,6 & 37,7 & 42,2 & $(39,9)$ \\
\hline 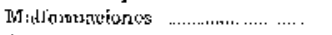 & $13, a_{1}$ & 19 & .15 & 14,4 & $25, \overline{7}$ & $(10,6)$ \\
\hline Anoxia & $4, \sqrt{1}$ & si & 3 & 23,8 & $\ldots$. & $(2, \bar{i})$ \\
\hline 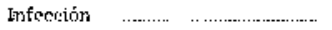 & 14 & 5 & $.72,2$ & $12, \mathbf{0}$ & 32.5 & $(24, \pi)$ \\
\hline 'litituma obstétrico ..... & 13,8 & $\{7$ & $-18,1$ & $\ddot{6}$ & 11,6 & $(7, b)$ \\
\hline Sifid]: & $\ldots \ldots$ & $\ldots$. & . & $\ldots$. & 8,3 & $(2,7 \pi)$ \\
\hline Porciento de piestituras ............ & 79,1 & $a 4,2$ & 71,8 & 68 & 58 & $(j 2,0)$ \\
\hline TOTAL INE MUERTES & 10.000 & 155 & 221 & 160 & $1.000^{r \times 4}$ & $(447,0)$ \\
\hline PORCIENT'O UL ALITCIPSIAS .. & 89 & 35 & $10 \mathrm{w}$ & 98 & $¥ 2$ & $(30,0)$ \\
\hline
\end{tabular}

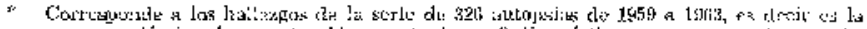

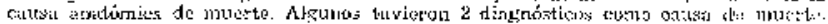

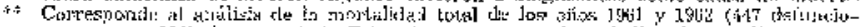

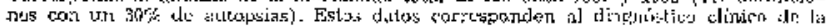

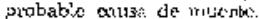

a: D Dhto extoximado. 


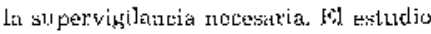
microstópico ha sufrido tiroth tén lomiLan:ores ber relación bon las condicisnes de tratzij) y tu inexperiencia.

De dis 326 oulepsias zilboilizadas, 192 nin̄os presentun un peso inforiol a $2.500 \mathrm{gm},(5 \% \%)$ ) y 134 (1n peso supe-

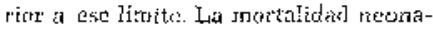
tal intrahospitalaria de hitestros Stryicios, revela un $72 \%$ de mños do peso bajis y un $28 \%$, de niv̆ros que jesan más de $2.500 \mathrm{gm}$.

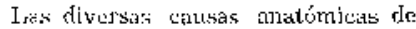
micrte se ha!jan e!ilyemlätizatdis' e th la Tablá I.

\section{ANATISIS WH LOS RESUIALDOS}

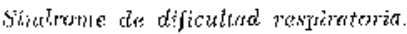
[l:L s:do casi exrlusivi áel promaluro.

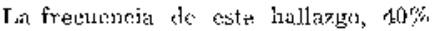

entre las altopsias renlizadas (Fig. 1), us mis o menos jæul que la cif a real de rontalidat por exta cilusa, $89,45 \%$ (Fig. 2). Un b:uet utimeru de niñus gut

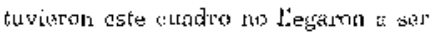
atrinpsades joor uria réativa falte de interés de ridlstro perronal para eon-

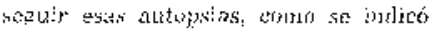
anteriomente. Dsto halläzón isilá en rialación ton el menor purceientio de premrituros ituturkiados.

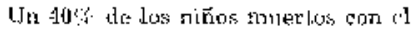
súndeme de dificulted respiratorial, prementarou la furmalán de meintoraJa h:al:tua. Jos restantes tuvicron ate-

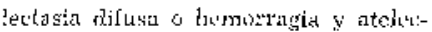
tasia pulmonar. 'I'ndus csoi casas vivicm Jon hures a pocus lías.

lin forma gencral, estos hallikgos sen vimilutez a !ns enconzliatos par otrijs atitorces ${ }^{3-1 ?}$.

\section{DIAGNOSTICO DE 326 AUTOPSIAS}

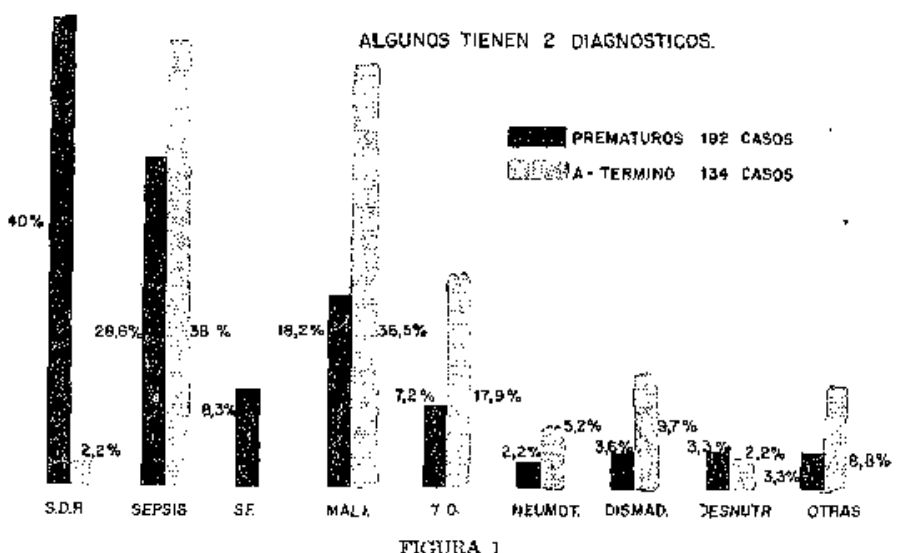

FIGUiRA ] 


\section{CAUSAS DE MORTALIDAD DE 433 CASOS}

\section{(30\% AUTOPSIAS )}

"Materalioad isiuno hyoka" 1.961- 1962

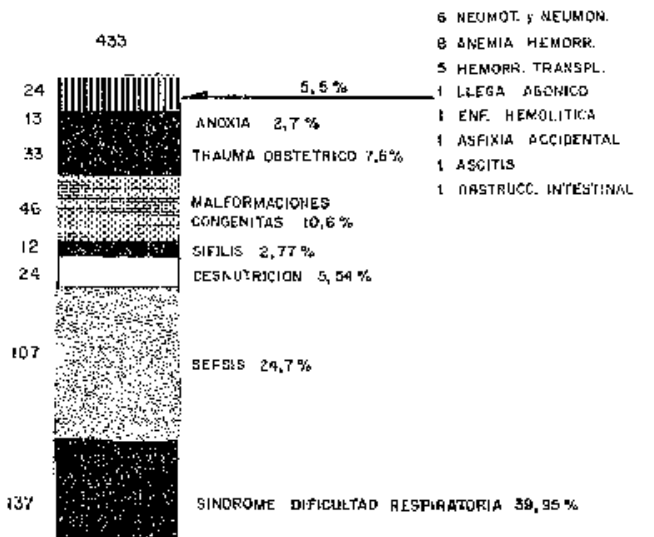

FIGCRA 2

La gran incidencia de prematuxez en nutstro medio (16\%) y la posibilislad de reducirla, como In han hecho sociedades más adelentadas ${ }^{1 \mathrm{j}-34}$, harla dismintuir et forma sistulicative la morbilidiad y mortalicad newnatal. Cuando le premeaturez disminuyc a un o u $8 \%$ dil mortalidad perinatal phedt:

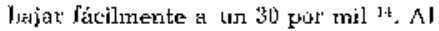
unismo tiempo que se disminuye la mortulidad, también st roduce el númoro de sujetos que por la premal.11- rez pronunciada desarrollan graves sccuelas 1t (retardo menta], convulsiones, trastomos de conducta, altericiohes serssoriales, etc.).

Alun frenle a esa incidencía buica de prematurez, si se presti una al:ención espeníl a la embaránada que tiene un l'iesgo alto en relación con 5 u producto, se puede consteguir reducir arin más lo prematurez y sus complitaciones, y otras causas de mortalidad neunatal. ${ }^{14}$. 
Infectones.-Un hallagro iresusite. de las autopsias ha sido le widencia d: infección: rue puede tieber sidu eatsa principal te muerte, o gue firte

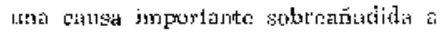
la caisa principal.

Entre los prematuros autopsiados, un 28,6\% (Fig. 1) presentarur: signos de infección; on un $20 \%$ la jntecturus sogurimente fur la culsa principal de nuerte.

Entre los nacidos a térmit!o : uldepsiaáos, un $38 \%$ tuvo hallazgos tvidentes de infeceión. El zav tuvo adensás otrì patología importinte que sogur anente fue la cause principal de muerte'.

Dc un lotal de 76 autopsiadss en manifestaciomes de ìfección hay un súmoro nayor de nacislos a término (5i). Hllos an su gran mayoria han si. do admitidos con infeccinties desarrolladas luera de la Matcrmidad, sm condiciones muy premanias y eon grave lesnutriıtón. Busos niños nue proseutaban elachros gustronteríticos, respimLorios, sepsis generalizadas, etc., no debian sur atendidos un La Matronidad. sinn en un hospital de niños. lis imperetivo que Jos hoopitales the la ciudad adcuen sus servicios para atender esos tasos.

fintre los 26 promatures antopsiados que tuvieron hallazos consistentes de scopis, la localización pulmo;ar fut muy troctente. En 5 hubo formasión Ie abscezos, En 1'2 luba una historia sugestiva de fnlecelún kdouirida isträuterinamonte; 9 de cllos murierun alstes de las 96 horas; 3 un las primexas 24 huras i'n lus chiss restatules, en que posithomento hubo infection ad aquirita extranterinamente, la muerte ocmrtít In mayoría de veces en ta segurtá semañ o despucs. Estc hullägo cs yimilar al encorblrado en atros Lugares ${ }^{t "}$.

Entre lus prematuros, en 2 cassul huho evidencia de meningitis (no se h:cieron estudios del terabro en todos los casos). En 2 tecsos hubicran signos de gustroenteritis. En 5 cases; hallazgns de infecciones generalizadus. Varits uutores haw publicado revisioncs de la inlección addouirida intraterinaruente: 2 , 7 is

In relación con la infección adquiri. da on cl ambiente axtralerino, lä condiciones muy difereisies an cue trabajannos, por la admisión de los casis. de iatectiones deserrollatios fuera de la MIalernidad, que en ntrias partes suz adruitidos en los hospitales de niñosi, so nos permilen hacer comparaciotes. [Dube seña]arse la gran Jimitación que? tenemos ch los estudios bacterindóngicos ex: nueztro Hospitinl.

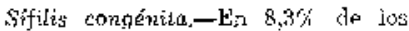
promaturos autopsiados hubo haltiygos de siflilis corbérila como causis de mucrte de egtos niños (Fig. 1). Estu altis inciclentia de silil:s entre los prena.wros autopsialos (no, la ihertes bueoytrado como causa eridente de muert un lus nacidos a túmino) podría demeistrar una frecuencia exagradamente a]ta de id siflil:s no tralacla entro là gestanles on nuestro medio, Fin tsLos casos ha hakido un interés milyo' en hacer esas autrysias. Cunito comparamos los hallizyess de autognias toon losi de ruestro djesnóstjeo clíuico (T'a13la I), esto rusulta mís apurente.

Los hallazgos de sífilis congénitúa en- 
tre los autopsiadss, hen sitho ya publiv cados 19.

Malfomaciones congénitas molures. --De los 81 casos encortidales con ma]. [otmaciones congénitas, \$1 łatr. sirdo macidos al térninu $y \quad 3.2$ prematuros. las ma]formaciones congénitas fuexon con frecuencia múlliples. En los rócej"los a tormituo, Jas malfurmueiuness cardiovasculares fuesty las más r:umerosas; les sigtuieron la.s dol sistcma nexvinso, digestivas, reudes, elc. 仯 tola. ción con hidroccófalias, micurotetialjiz:; cter, se ha ofcetuado otro trahajo dr estudion y revisisión ${ }^{20}$.

Entre los preminturos, l.s. malfo:maciones digegtivas Jucron lạs más fre-

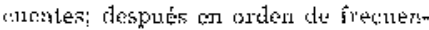
cii vinipron las merviosars, respiratoriak, cartiovasculares, ete.

En nuessiral exisuístícil de maliormaciortes, constan í casos de nongolismo que tuvieron graves rualfurmaciono: que produjeron la mucrte; 3 casos the hiperplasia congénita vle supratronales en seudohermafroditas femenivos mas-

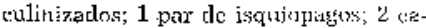
:os de osteogénesjs imporfecta con 「racturas múltiples intraútero: "2 xisos de síndrome de Turner; elc. Las mallurmacirmes congconitas entre Ios reciên wageidos, sts maleria de entudio en otres puatblicactomes 21-24.

Trauma ohstétrico con henomresfits.

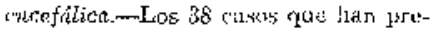
rentado esta patolngín (24 aicidos a léruino y 14 prematuros) no revclan lit frecuencia real do esitat patolosía; csprejumente si se considera la gran incitloxeia de sepsix que hace apareces menures a Ias otras chusils de ruuerte.

Se puede enmprender como, las cundiciones socio-económicis y culturates de las gutstantes, hocen mán rlifîil ol rontrol prenatal adceuado y la prevejtción del traume sbstestrice.

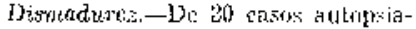
dos que presentaron un eundro de dis-

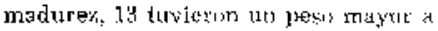
2.500 gramos, y 7 un peso inferior al ese linte. Wo atres elios, $72 \%$ thlvirdorn un cuadro pulmonar (aspira-

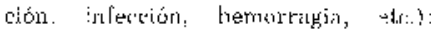
$28 \%$ tuvieron treuma obistétricn F, ? 62\%: de estos nifios fuexon bijos de primíparas.

Las otras causas de muerte quo cunstin en el cuadro, no hay sicte anajizadas.

\section{IJISCUSION}

Lil somparación de los valures oblor. rjidos en el presente estudicr, con las de otros lugares (Tabla I), hechas tis silveclades en relación con las condiciones particulares de nluestro medio, demuestran la alta freruencia de infocciones y la incidereial alta da siffilis congénila.

Un estuctio más cumpleto y sistoná-

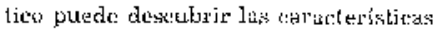
de la patología neonatal de nucstro meitio.

No se puede pretenter detormina: pot los estudios ratajzadus, en espectal por In calidad de ellos, una información esstadistica calre permitá hacer comparaciones prectisas cort la patcolngia neonatal de otros medios. Iintre 
nuestros hospitiles se destaca ol Sexwcio de Podiatría do di MLatern:dad "Isidro Ayor:a" por su interés cel reilizar estudios histopatológicus y su ufítu tc botener una informacisóts estáclística de la mork; Fidak y mortakidud en lis ax:takiss momentos.

\section{RESUNFN}

l'n itirlad "Isidro Ayora" de Quito, licutadsr, se hal realizadu el $: 30 \%$ do a: sivk (incompletas) atc las muertes neo. natales intrabosyitulaxias. Do la revisión y antilisis dt: los resultados obtenidus se concllyye aude ol índice do mortalidad noonated intruhospitalistia es 29 por mil $y$ el de mertislidad perriretal es $\mathbf{4 9}$ por mil.

Ex nlarmante, la gräni incicleneia de inletciones por la admisión de retiér! naciclos infcetados a Los Servicios dc Pediatrìi de lá Maternictra, y por :a fa]. ta de cujdaco inclividual en las salas de niños.

Asimismo se cncontró muy elevalta lis intidencia de siffilis, en cuyos casos mucetre morsonal dernustró pralliculiav interés para realizar las autopsias resvettivas.

En l'azón do las circunstarcias, ostast: de liempo del persomal mético incexperiencia, la información obtenida ru purmile Hinter un bues anúlisis de la s:tuación local, ni cumparationes witikg con las de otros howpitalos.

\section{SUMYARY}

In: five yeats (1959-1963) at the Mis1.erlity "lsidro Ayore", Quitito, Ecuildor, 30 \% of the neronallal deathis biave hatd inciumplete autopsess.

A renuatol mortidity of $24 \mathrm{sic}$ and ì periun?tal mostality of 49 \% have befen "eportcod from thial hespital in the vears I.9tit-1962.

'Thl, iargo incidence inf infections because if the admission of infested newborrs: to the Maternily and the Iack of indivilual rare of the jillapts: are presented.

The incideace if syyjhilis and the perticular inderest of our persnma' to nertorm the poxtmorters examinations in cases of congenital metfurm:ations, coulgetital syphilis and other particislier pellholngy are analized.

Ecounse of the vircunstaneci; inexperience and shortuge of litue the cata doosn't pernit a sood :malysis of the lexesl siluation to sompare wilh similar experiemties of other hospitals.

\section{HEFLIRHCNCIAS FIBLIOCMAEICAS}

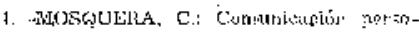
n.e.

2. ESPIRUSA, N., NARANJO, C.: Come!:L-

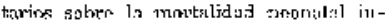

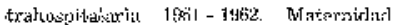

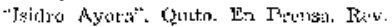
Fim 1axi. Irdiat.

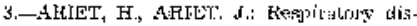
orders of the newhen infart. Telial Clin. N. Am., 59-6.1, 1957.

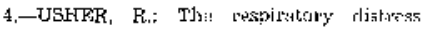
s.potrome as promaturity. Ped. C.s. N. Am., 525-538. 1961. 
:- 1月iklscole, S., SuIITE, C.: Nooxical

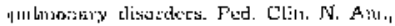

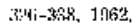

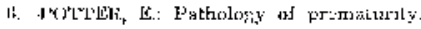

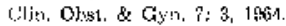

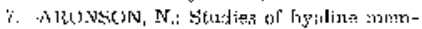

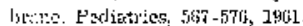

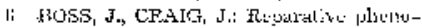

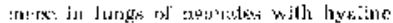

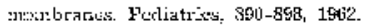

1) MIII,ER, H.: Reupiratury dïlrus syn-

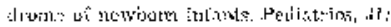
!ifit-579, 19tis.

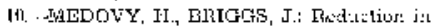

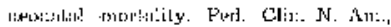
쌔, 195.5\%.

11.-TO'Ll'Ek, E.: Putholosity of the futus and Whe newbor's. 'l'ke yers' hook yali!, sher's, Irc., Heptinicd, ]Yöil

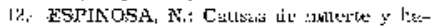

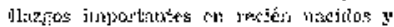

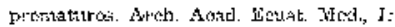
$26-43,1960$.

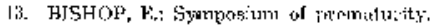

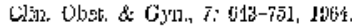

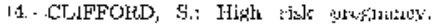
Niew kin., J. MIod. ¿t]: 5, 1964.

].5.--HLARDYMLNT', A.: Costrol of inrectiono

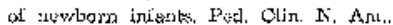
$287-298,1958$.

16. HACATRTY, R.: Bnctermal Infartions in thes asvibs:ri, Ptel, Clis. N. Akx, 461--191, 1961.

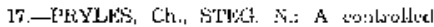

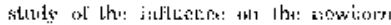

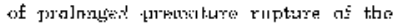

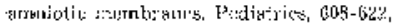
$190 \%$.

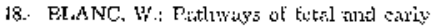

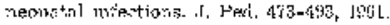

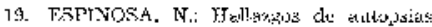

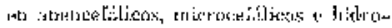

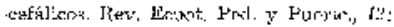
127-13B, 1064.

20,-ESHINUSA, N.; NALRNJU, C.: ZUENO,

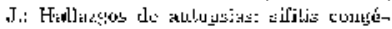

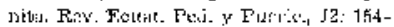
163, J界1.

2t.- VICCHJ, M.; MRtAS, G.: Kiñúa rou'-:-

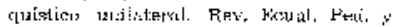
P1uric., J2: ]'s', 1961.

22. ARIAS, G.: Maifotmacioms corpenitas

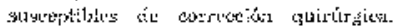

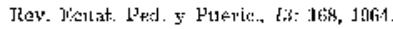

23. -AIRIAS, Cx.: Liguino amaniotico y patolo-

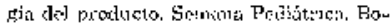
getá, Co'onshin, kgostn, lllow.

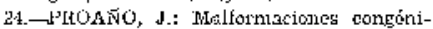

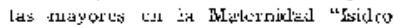
Ayom" cle Quito. Tesis Dooto:il. Univernildad Cétettril dê Quito. 